\title{
Inception and Development of the Papanicolaou Stain Method
}

\author{
Nikolaos Chantziantoniou ${ }^{\mathrm{a}}$ Amber D. Donnelly ${ }^{\mathrm{b}}$ Maheswari Mukherjee ${ }^{\mathrm{b}}$ \\ Mathilde E. Boon ${ }^{d}$ R. Marshall Austin ${ }^{c}$ \\ aDivision of Anatomic Pathology, Department of Pathology, Sidra Medical and Research Center, Qatar Foundation,

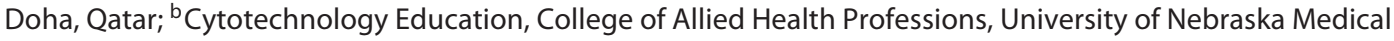 \\ Center, Omaha, NE, and 'Pathology Department, Magee Womens Hospital, University of Pittsburgh Medical Center, \\ Pittsburgh, PA, USA; ${ }^{d}$ Boon Institute, Lieveren, The Netherlands
}

\author{
Keywords \\ Vaginal fluid smear - Papanicolaou stain - Pap stain . \\ Cornification - Fixation - Pap test
}

\begin{abstract}
Objective: Cytodiagnoses of specific malignancies are enabled through analyses of abnormal nuclear chromatin and cytoplasmic features in stained cells. Aim: The objective of this work was to explore the inception, development, and chemistry of the Pap stain method introduced in 1942 by Dr. G.N. Papanicolaou. Study Design: To achieve this, we carried out a review of the English literature. Results: Between 1914 and 1933, Papanicolaou first analyzed vaginal squamous cells in guinea pigs and later in human vaginal fluid samples using hematoxylin and eosin with limited color reactions, correlating the cell-type morphology with endocrinology and histology. The 5-dye Pap stain method evolved through 2 salient phases. The first, between 1933 and 1942, saw the introduction of alcohol-ether fixation and aqueous waterblue staining to enhance cellular transparency, aiding the distinction of cervical cancer cells from benign cells, with quantitative and qualitative assessment of squamous cell maturity. The second phase, between 1942 and 1960, saw the introduction and refinement of various alcoholic cyto-
\end{abstract}

plasmic counterstaining schemes with orange $G$ and EA (light green, Bismarck brown, eosin) and phosphotungstic acid, allowing wider ranges of polychromasia and further enhancing cellular visualization, facilitating the distinction of cell types and improving diagnostic confidence. Conclusions: Development of the Pap stain method followed specific historical and scientific events. The staining method evolved following incremental improvements in cellular transparency achieved through tailored cellular fixation and cytoplasmic staining using variable dye and $\mathrm{pH}$ combinations.

c) 2017 S. Karger AG, Basel

\section{Introduction}

A cytologic diagnosis of malignancy is fundamentally based on the analysis of abnormal nuclear chromatin patterns in stained cells. The assessment and grading of nuclear abnormalities along with cytoplasmic morphology are the hallmarks by which malignancy and cell lineage may be cytologically determined. These diagnostic principles matured through extensive research and testing for their clinical relevance in parallel with incremental chemical enhancement of cellular transparency through tai-

\section{KARGER}

(c) 2017 S. Karger AG, Basel

E-Mail karger@karger.com

www.karger.com/acy
Correspondence to: $\mathrm{Mr}$. Nikolaos Chantziantoniou

Department of Pathology, Sidra Medical and Research Center PO Box 26999

Doha (Qatar)

E-Mail nchantziantoniou@ sidra.org 
lored cellular fixation and staining. Among the major achievements in the history of cytopathology, the eponymous Pap stain method formalized by Dr. George N. Papanicolaou in 1942 was foundational $[1,2]$.

This review explores when the Pap stain method was conceived and what prompted its ongoing development. Of particular interest was the development of the stain's unique polychromatic attributes. We studied Papanicolaou's publications, seeking insights into his design and refinement of the Pap stain method.

The biochemical and physical properties of the Pap stain's constituents have been studied extensively by Boon and others [2-4]. Specific citations document historical timelines and the development of Pap stain fundamentals.

From the English literature, the evolutionary phases of the Pap stain followed a series of historical and scientific events which primarily occurred after Papanicolaou first noticed cervical cancer cells in 1925 [5]. After introducing the 5-dye protocol in 1942, he modified it formally on 3 occasions. These were documented in Diagnosis of Uterine Cancer by the Vaginal Smear in 1943 [6], Atlas of Exfoliative Cytology in 1954 [7], and a supplemental note for the Atlas in 1960 [7]. These modifications reflected his experimentations to continuously perfect the stain and its color reactions up until his death in 1962 [2, 8, 9]. The final version of the Pap stain technique facilitated clear visualization of nuclear and cytoplasmic cytomorphology through cellular transparency. This allowed assessment of key cellular features, including: nuclear chromatin alterations; cytoplasmic structures in maturing squamous epithelial cells; degrees of cellular degeneration or necrosis, and production of mucin, glycogen, and keratin. By revealing these elements, Papanicolaou developed diagnostic criteria distinguishing the cytopathology of cervical squamous cancer and adenocarcinoma. Cellular features were then illustrated through remarkable water color drawings by the scientific illustrator Hashime $\mathrm{Mu}-$ rayama in Papanicolaou's 1943 and 1954 bound texts [2, $6,7,10]$.

This review incorporates excerpts from Papanicolaou's publications - some of which are extensive. This is intentional to catalog literature dating back to 1917 that is often not easily available to practicing cytologists. We include notations with anecdotal information put forward by the authors. The research and historical events that led to the inception and development of the Pap stain method are not only cytologically significant, they also facilitated wide-reaching contributions in modern diagnostic and preventive medicine [10].

Inception and Development of the Pap Stain Method

\section{Dedication}

This work is dedicated to the International Academy of Cytology, which in 2017 is celebrating its 60th anniversary since its founding in Brussels, Belgium, in 1957 with Dr. G.N. Papanicolaou as its first Honorary President, and to Acta Cytologica, which was also coestablished in 1957.

\section{4}

In September 1914, Dr. Charles R. Stockard hired George Papanicolaou as an assistant in the Department of Anatomy at Cornell Medical College, New York. Stockard was chairman of the department and an experimental anatomist studying the effects of alcohol on guinea pig chromosomes and their possible transmission to progeny. Shortly after his arrival at Cornell, Papanicolaou was tasked to extend his previous $\mathrm{PhD}$ research work on sex determination and chromosome structure in Daphnia fleas, to guinea pigs. In November 1914, Cornell allowed Mrs. Andromache Papanicolaou to work alongside her husband as a voluntary technical assistant $[2,8,10,11]$.

Papanicolaou tested the theory of sex determination by $\mathrm{X}$ and $\mathrm{Y}$ chromosomes in spermatozoa and ova. His study involved harvesting and analyzing ova from experimental guinea pigs during chromosomal division and ovulation. This meant animal sacrifice at a specific time, which was practically impossible for him to determine without knowing the estrous cycle parameters of these lower mammals. As Papanicolaou wanted to study ovarian function, physiology, and the long-term effects of hormones, he preferred to track ovarian function in living animals, favoring the surgical removal of ovaries. Paradoxically, this also required the precise timing of ovulation. The inconspicuous estrous flow in these small animals posed multiple dilemmas $[8,12]$.

Papanicolaou considered the impasse. He knew these mammals menstruated, but only feebly. He had to reveal the onset of the guinea pig menstrual cycle and its completion to pinpoint ovulation. He supposed 1 approach might be to collect and analyze vaginal fluids over a period of time for clues to the timing of menstruation. This hypothesis led him to a local medical supplier from whom he obtained a nasal speculum. His plan was deceptively simple: insert the speculum into the vaginas of these small animals and with vault dilatation aspirate identifiable fluid using a thin pipette; thereafter, analyze fluids microscopically for predictable correlating characteristics $[8,10]$. 
Using this method, Papanicolaou collected miniscule fluid samples from the experimental animals daily. $\mathrm{He}$ spread the fluids thinly onto glass slides and stained the smears with hematoxylin and eosin (HE) stains, keeping meticulous notes of the fluid characteristics over time. From detailed study, Stockard and Papanicolaou described the ovulation and menstrual cycling of guinea pigs precisely by analyzing epithelial cells against their background characteristics. They published their findings in a 3-page report in Science in 1917 (entitled "A Rhythmical 'Heat Period' in the Guinea-Pig") [13].

Intrigued by these discoveries, which correlated vaginal cellular changes with ovarian function, Papanicolaou followed through on his intuition to republish their findings but in considerably greater scientific detail. He became enthralled by the exfoliated epithelial cells entrapped in fluids and their potential in reflecting the underlying physiology. He expanded upon their data and produced his first discussion of the staining methodology coupled with crude photomicrographs of epithelial cells and respective uterine histology. Coauthored with Stockard, Papanicolaou's efforts culminated in an elegant 58page report published in the American Journal of Anatomy in 1917 (entitled "The Existence of a Typical Oestrous Cycle in the Guinea-Pig - With a Study of Its Histological and Physiological Changes") $[2,14]$.

Papanicolaou's work facilitated the precise timing of guinea pig ovulation through analysis of desquamated epithelial cells arising from 4 cycle stages. He described nuclear and cytoplasmic features, and their physiologic context [14]. The following extract is from Stockard and Papanicolaou (1917) [14]:

By the use of such a simple method, it was readily determined after examining the first lot of animals for a few months that a definite sexual period occurs lasting for about $24 \mathrm{~h}$ and returning with a striking regularity every fifteen or sixteen days.

The concept of a simple method of vaginal fluid collection and microscopic analysis emerged documenting the possibility of cytologic interpretations through vaginal fluid analysis $[2,8,10]$. In subsequent passages, Papanicolaou detailed distinctive epithelial cell features observed with HE staining [14]:

The cellular character of a smear made at a given stage differs from the cellular make-up of all other stages. The relative numbers of various cell types in the fluid at different stages are so definite that one with a little experience may diagnose the exact sexual stage of the animal concerned solely by an examination of the smear.
The mucous fluid is seen to contain an abundant mass of cells which, as shown in the figure, are of a squamous type with very small pycnotic nuclei sometimes broken into pieces. The cell protoplasm is also greatly degenerated having only a weak affinity for the plasma stains and exhibits a reticular structure. These cells derived from the wall of the vagina characterize by their presence and great superiority in numbers this first stage.

There are, however, to be seen particularly toward the end of the first stage a certain number of elongate, cornified cells without nuclei, which are desquamated from the more external portions of the vagina. These cells contrast in appearance with the first type cells since in smears stained with haematoxylin and eosin they present a decidedly red color, while the abundant first type cells are almost grey. The red cells rather serve to indicate an intermediate period between the first and second stages or periods of the flow, and may really be found during both stages but particularly at the end of the first and beginning of the second stage. In addition to these two kinds of cells other types may also be found in a first stage smear but they never present in such abundance nor are they so typical as the two just mentioned. All of the cells float freely in the mucus without assuming any definite arrangement.

During the second stage the vaginal fluid is filled with enormous numbers of cells which cause the cheesy-like consistency of the discharge at this time.

The nuclei are fairly well preserved showing only slight signs of degeneration. The protoplasm has not greatly deteriorated and gives a good staining reaction thus differing from the grey-staining first stage cells. The cells are present in innumerable quantities forming the thick cheesy substance while the mucous secretion diminishes more and more until it almost disappears. This stage is of short duration.

The third stage begins with the liquefaction of the cheesy mass. A microscopical examination shows that the cells of the second stage become less and less numerous, while a great number of polymorphonuclear leucocytes appear among them.

When the end of this process is reached almost every one of the cells has become isolated from others of its kind and lies in the midst of a number of leucocytes.

The fourth stage shows the same condition as the preceding but often at this time a slight hemorrhage takes place, though this does not always occur. A microscopical examination of the hemorrhage fluid shows in addition to the great number of red blood corpuscles, a large number of leucocytes and also desquamated cells of the second stage, some of which are penetrated by leucocytes.

Throughout the first week after heat the little fluid which exists in the vagina contains chiefly leucocytes and a few atypical desquamated cells. During the second week the number of epithelial cells increases more and more and among these atypical cells there may exist isolated cells of the first or of the second stage type.

At the fourteenth and fifteenth day the number of first stage cells already described begins to increase gradually and the growing proportion of these cells indicates the approaching new period of heat.

Papanicolaou recognized complex associations between ovarian function and epithelial cell biology. $\mathrm{He}$ achieved this by analyzing cellular affinities for stains, assessing patterns and color intensities from the limited hue variations possible through bichromatic $\mathrm{HE}$ staining. $\mathrm{He}$ graded degrees of cornification from intensities of red 
color, and cellular viability from gray color. But it was nuclear pyknosis and fragmentation in poorly staining cells that ultimately influenced the development of the Pap stain. Papanicolaou thought these phenomena represented degenerative processes that predisposed the resulting stain reactions.

Whether smeared epithelial cells were fixed for the 1917 guinea pig experiments remains unknown. The various degrees of cellular degeneration, nuclear and cytoplasmic fragmentation, and the color contrasts noted between noncornified and cornified cells led Papanicolaou to conceptualize a spectrum of cellular physiological processes stemming from cyclical ovarian endocrinology and the effects of inflammatory cells. He introduced the following terms to describe his observations: "degenerated" and "atypical." These terms implied detection of subtle yet distinct cellular changes. Through the term "atypical" he intended the communication of unusual cytomorphology, differing from that of other cells of the same type.

1919 became a pivotal year, marked by the death of his father. Due to his strong sense of obligation and selfdiscipline, he reacted to sadness and discouragement by delving with even greater determination and singlemindedness into his scientific endeavors $[2,8]$.

\section{0}

In 1920, being also a physician, Papanicolaou questioned whether or not guinea pig epithelial cell changes and estrous stages might also occur in human females. However, not being licensed to formally practice medicine in New York, he had no access to volunteers to apply his experimental vaginal fluid smear method [2, 8-10]. Astonishingly, Papanicolaou approached his wife, Andromache, and she consented to his taking vaginal fluid samples from her daily using the same collection technique. This exercise commenced in 1920 and ended 21 years later when Papanicolaou formally reported the refined 5-dye Pap staining method in 1942 [2, 11].

Along with ongoing research in guinea pigs, Papanicolaou then analyzed epithelial cells collected solely from Andromache. As she remained nulliparous throughout her life, he studied cells reflecting regular ovulatory cycles and unchanging bacterial flora with gradual physiologic changes as she approached menopause. It was from her samples that he developed a foundation of benign human vaginal epithelial cell morphology. His meticulous study established baseline cytomorphology correlated with ovarian function and human aging in the absence of dis-

Inception and Development of the Pap

Stain Method ease. This robust knowledge of usual, or typical, cytomorphology was to prove invaluable $[2,15]$.

In early 1925 Papanicolaou embarked upon a key project analyzing samples collected from other consenting women visiting the Woman's Hospital in New York under a formal arrangement brokered by Stockard. This affiliation brought him into the clinical setting $[8,10]$. He then studied human pregnancy, endocrinological rhythmicity relative to ovarian function and, eventually, the epithelial cell alterations arising from cervical neoplasia. Importantly, he also studied various vaginal bacterial types and infections and their unique effects on epithelial cell viability and staining. Through this work, Papanicolaou revised his understanding of epithelial cytomorphology. These were key underlying factors in the eventual development of the Pap stain.

It is noteworthy that between 1917 and 1924 Papanicolaou published 12 papers to report findings from endocrinological research in guinea pigs and other mammals. It was not until 1925 that his first descriptions of diagnostic studies in women appeared in the Proceedings of the Society of Experimental Biology and Medicine (in a report entitled "Diagnosis of Early Human Pregnancy by the Vaginal Smear Method") [16].

Papanicolaou noticed malignant cells in smears prepared from patients treated for cervical cancer in 1925. This revelation set a defining course for him and cytopathology. A new era emerged with a realization of the diagnostic potential harnessed in vaginal fluid analysis. Nevertheless, cervical cancer cells and their unique cytomorphology through HE staining startled Papanicolaou. We believe these specific events in 1925 catalyzed the inception and incremental development of the 5-dye polychromatic Pap stain protocol $[2,8]$.

\section{8}

By 1928, as Papanicolaou felt he had assumed sufficient experience and confidence in his cytologic method's utility in detecting subclinical cervical cancer, he summarized his conclusions in a public presentation entitled "New Cancer Diagnosis," delivered with enthusiasm in 1928 during the Third Race Betterment Conference in Battle Creek, Michigan. A short summary of his presentation appeared in the conference's proceedings $[8,15,17]$. The following is from Papanicolaou (1928) [17]:

Another factor that makes this work a little more difficult in the human is the tremendous variety of bacterial forms that are present in the human vagina; the number is much higher than that of 
the bacterial flora in other mammals. In fact, in every case you may see very different flora, and this may be associated with various morphological conditions.

For this reason I found myself compelled to undertake some pathological work, because otherwise it was impossible to know what was normal and what was pathological and how far these various bacteriological forms were pathological changes. So I studied smears from various pathological cases, including all kinds of infections... and finally cases of benign and malignant tumor. One striking fact was found in all cases of benign tumors, or other pathological conditions, and of the normal. The changes that occurred were all a little different from what happens in cases of malignant tumor.

If we take, for instance, as an example, one case of carcinoma of the cervix, which is the most common type, in the smear you may find some cancerous [mistyped as "conscious" in the proceedings] cells. When the cancerous [again mistyped as "conscious"] cells are present - and they are present in most of the cases - they are easily recognized, much easier than in sections, because in sections the cells are crowded one against the other and you do not see the quality of the cell; whereas in smears the cells are isolated and the morphological changes that occur in these cells are really so different but the recognition of the cells is not so difficult. It is not an exaggeration to say that certain cases of carcinoma of the cervix may be diagnosed by the presence of only one of these cells.

The changes of the cells are mainly an abnormal growth and the development of the nucleus of the cell. The nucleus becomes very large, out of proportion to the cell, and becomes very dark and dense, and often it shows fragmentation, it breaks up in pieces. This is a very common occurrence.

Papanicolaou did not mention fixation or staining practice in the proceedings summary. We nonetheless suspect from the published photomicrographs that cells may have been alcohol fixed given their sharp nuclear outlines [17]. Perhaps discussion of staining methods at that point seemed less important to Papanicolaou than the novel detection of cancer cells. His intended message to the medical community was that, through the cytologic method, cervical cancer could be reliably identified potentially at an earlier and more treatable stage of development in asymptomatic women.

These promising developments stalled as Papanicolaou's Battle Creek presentation failed to capture the support of his peers; neither gynecologists nor pathologists, accustomed to classifying cervical disease through biopsy, were supportive. The notion of medically binding diagnoses rendered on the basis of colored, desquamated, senescent epithelial cells trapped in vaginal fluids, and smeared on glass slides, was exceedingly unorthodox. Superimposed upon these setbacks was also the onset of the Great Depression in 1928, posing substantial socio-economic priorities. Facing a bleak reality, Papanicolaou felt deeply discouraged and, despite his nature, dismissed his study of the vaginal smear method for diagnostic purposes. Utterly rebuffed, he might have abandoned his research work altogether had it not been for Andromache's intuition and encouragement, which kept him engaged in cytologic investigation of the hormonal cycle $[8,11,18]$.

These events launched what some have referred to as Papanicolaou's "lost decade" in cytopathology. However, his bibliography of publications and presentations produced between 1928 and 1938 suggests otherwise [2]. Even though his work on cervical cancer diagnosis had ceased, it was a decade of major discovery and development, as revealed by his pioneering reports on human hormonal cytology, coauthored with Dr. Ephraim Shorr. Shorr was an endocrinologist at the New York Hospital and a dynamic researcher [8]. Ironically, it was from the work in the earlier part of that "lost" decade that the Pap stain method emerged.

These collaborators developed novel practices to manage patients with complications of menopause and infertility by analyzing stained epithelial cells in vaginal fluid. Through Shorr, Papanicolaou gained invaluable knowledge of the human sex hormones and their effects upon vaginal epithelia and exfoliated cells. Yet, in the background, cervical cancer cells troubled him. One problem was their bizarre nuclear morphology when compared to normal cells. Another was cytoplasmic cornification and its variable intensities, and how cancer cells naturally dislodged in clusters in larger groups, making individual cells difficult to see. Also challenging were the abundant infiltrations of inflammatory cells along with profuse blood, and obscuring necrotic material in cases of advanced disease. The degree of squamous cell cytoplasmic cornification, apparently variable even within a single patient over a course of time, was another challenge. Cervical cancer cells had intense and extensive cornification obscuring their nuclei, much unlike normal cells.

The nuclei of malignant epithelial cells were hyperchromatic, opaque, and fragmented into pieces. However, more concerning to Papanicolaou was that he had seen fragmented nuclei in Andromache's cells, in guinea pig cells, and in cells from women with florid bacterial infections and inflammatory reactions. His inability to satisfactorily explain these observations was unnerving. $\mathrm{He}$ had to bridge these gaps with workable tools to arrive at clinically sound interpretations. He needed a better definition of normal cellular morphology to differentiate subtle alterations reflecting hormonal fluctuations from neoplasia, and to distinguish pathological cytomorphology from the broad spectrum of normal morphology seen in Andromache's samples. Papanicolaou foresaw the 
need to simplify and standardize his methodology to enhance its reproducibility; otherwise, the vaginal smear method offered trivial diagnostic and clinical advantages over biopsy - as was alleged in the wake of his 1928 Battle Creek presentation [8].

\section{3}

Papanicolaou's remarkable work to overcome these problems emerged in a seminal paper published in 1933 in the American Journal of Anatomy (entitled "The Sexual Cycle in the Human Female as Revealed by Vaginal Smears") [5]. The report consisted of 118 pages documenting the problems in reconciling cellular features in both healthy and diseased women with those observed in guinea pig cells. Papanicolaou's discussion focused on the desquamation, morphology, and presence of cornified and noncornified cells, and their association with bacteria, inflammatory cells, and menstrual cycling.

Papanicolaou cited the work of Ramirez (1928) and Moser (1928) [5]. These scientists studied the nature and structure of the vaginal squamous epithelium, and the variable cornification of cells comprising the stratified epithelial zones. Ramirez used the Leishman stain (a mixture of methylene blue and eosin) and likewise noted the reticular granularity of both the nucleus and cytoplasm. Moser used Delafield's HE, commenting on similar observations. Papanicolaou noted Moser's use of air-dried epithelial cells prior to staining. He also acknowledged similarities between his findings and those of his peers, speculating that air drying was the root cause of nuclear opacity and fragmentation, not biology. $\mathrm{He}$ thus resolved that $\mathrm{HE}$ staining was unsatisfactory for human vaginal fluid samples mainly due to their potential cellular and bacterial complexity. His decision justified deviation from conventional staining practices. When exactly Papanicolaou experimented with alcoholic fixation remains unknown, and it may have been prior to 1928 (as discussed above). Nonetheless, he provided the following defense for eliminating the air drying of smeared cells. The following is from Papanicolaou (1933) [5]:

Most of the smears which I examined up to February, 1925, were obtained from a single human case (special case). This was an ideal case to work with, because of perfect regularity in the menstrual periodicity and a complete lack of any serious disturbance or of any bacterial contamination. It has been by far the most normal and typical case that I have had an opportunity to study. Several smears obtained during this first period from the clinic of Cornell Medical College in New York City, through the courtesy of Dr. John McGrath, were taken mostly from pathological cases. These have been little utilized for the study of normal standards.

In February, 1925, a valuable association was established with the Woman's Hospital of New York City. This was rendered possible through the cooperation of Dr. George Gray Ward, director of the Woman's Hospital, Dr. Charles R. Stockard, administering a grant from the Committee on Problems of Sex of the National Research Fund, and Dr. Robert L. Dickinson, representing the Maternal Health Committee. A special assistant was assigned to this work, whose technical skill in the preparation of smears has been extremely valuable.

Our first effort was to obtain a sufficient number of control smears from normal cases. We selected twelve healthy young women, the majority from the personnel of the hospital, and arranged to obtain daily vaginal smears through several menstrual periods without interruption. Later, the material was extended to include smears from pregnant women who came to the clinic of the hospital for examination, and also from various pathological cases treated at the hospital.

From the start of this work at the Woman's Hospital up to the end of 1929, more than 900 cases were examined. Almost every important pathological condition has been more or less thoroughly considered. There have been smears from cases of pregnancy, abortions, threatened abortions, ectopic pregnancies, various infections or inflammatory processes of the genital tract, cystic ovaries, pus tubes, and various benign and malignant tumors of the ovaries, uterus, cervix, vagina, and mammary glands. There were also several cases of sterility due to various causes.

This study of pathological smears has helped in an understanding of normal processes and opened a new possibility for diagnosing various gynecological conditions. In a preliminary note (1925), I referred to the application of the vaginal smear method as a guide in the diagnosis of pregnancy and of pathological conditions.

In the last few years attention has been given to the characteristic smear changes which seem to accompany cases of malignant tumors of the genital tract. A preliminary report on these observations was given at the Third Race Betterment Conference (1928). Several types of abnormal cells with enlarged, deformed, or hyperchromatic nuclei are present in such smears. Leucocytes are numerous and display a high phagocytic activity. Mononuclears become especially prominent. Erythrocytes are commonly present. A more detailed discussion of this phase of the study will be given in a subsequent publication. In this paper we intend to describe only the normal smear findings and to discuss their possible application in a detailed analysis of the human sex cycle.

The technique used in these studies has been rather simple. In dealing with human material one benefits greatly by simplifying methods and avoiding unnecessary technical difficulties. In order to obtain the samples of vaginal fluid a glass pipette 8 inches long and $3 / 8$ of an inch in diameter was used. The pipette was slightly curved at the end. The opening was small and a strong rubber bulb at the opposite end was used to produce suction in collecting the fluid. The sample of vaginal fluid may be taken with the aid of one of these pipettes by a technician or a nurse. Some of the patients were able to take their own smears. The sample is obtained from a region 5 or $6 \mathrm{~cm}$ within the vagina and is spread on a glass slide as a heavy film, much heavier than an ordinary blood smear. Soon after the spreading and before the smear has dried the slide is placed in a jar containing equal parts of $95 \%$ alcohol and ether for quick fixation. When ether was not available, plain alcohol was used. 
Proper fixation of the vaginal smears before drying is important, although this has been somewhat underestimated by most of the workers in the field. A smear which has been allowed to dry either before or after fixation may serve for the simple recognition of a typical stage, as has been done on rodents, but is not suitable for a detailed cytological study, especially in the human. The cells, as well as all other elements, are affected by the drying and lose their true appearance. Several investigators have probably failed to recognize certain morphological characteristics because of the use of dry smears.

The slides were kept in the alcohol-ether fluid for from half an hour to $1 \mathrm{~h}$, but smears may be kept in this fluid for several hours or even days without much harm. After fixing in this way the slides are carried through 80,70 , and $50 \%$ alcohol into distilled water as preliminary to staining.

The staining of vaginal smears with haematoxylin-eosin gives good general results and a fair differentiation of the cornified cells, and such a stain may be considered as very suitable for the recognition and study of the fundamental morphological changes in the smear of rodents. This stain, however, in the study of human smears, did not give satisfactory results.

After using various staining fluids, I succeeded in developing a simple stain, consisting of a combination of haematoxylin, eosin, and waterblue, which seems to offer certain advantages for the general staining of vaginal smears. This secures a sharp outline of the various cell types with a variety of shades from an intense blue to an eosin-red. The cornified cells, which are almost constantly present in the vagina, take an intense eosin color and are sharply differentiated. On the other hand, cells derived from the deeper vaginal layers, which are free from cornification, are stained strongly blue. Partly cornified cells show various shades of purple. Superficial secretory cells, containing mucus, display a typically characteristic bluish-purple tone. Leucocytes, as well as bacteria, are also sharply outlined. Eosinophils show distinctly red granulation. $\mathrm{Mu}$ cus takes a bluish or purplish shade. The contrasts are much sharper than with ordinary haematoxylin-eosin and the cornified cells are particularly differentiated. This is important for the vagina, in which the cornification is very pronounced, especially during certain periods of the cycle.

The exact method is as follows: slides are first stained with Ehrlich's haematoxylin for about $5 \mathrm{~min}$, then carried into running water for at least a quarter of an hour. After this they are stained with a $0.5 \%$ eosin for 3-4 min, rinsed well in water, and finally stained for $1 \mathrm{~min}$ in a $0.5 \%$ solution of waterblue. Following this they are rinsed well in water and then carried through 50,70, 80, 95\%, and absolute alcohol into xylol and mounted in Canada balsam. The stain is permanent and does not fade easily. Several other methods have been used, but none was as satisfactory as the above.

The Pap stain protocol then consolidated immediate fixation of vaginal fluid smears in an alcohol-ether mixture of equal proportions followed by aqueous hematoxylin, eosin, and waterblue staining to facilitate nuclear and cytoplasmic contrasts. Papanicolaou defended the "simple" stain's color reactions as they enhanced cornification with sharp cellular outlines in squamous epithelial cells and better reflected their maturity. Based on the numbers and types of squamous cells observed, he raised infer- ences as to the degree of squamous epithelium maturation and the presence or absence of circulating hormones. The following is also from Papanicolaou (1933) [5]:

The topographical origin of the various cells encountered in the vaginal smears is not always clear. It is practically impossible to link every cell with the zone from which it arose. One may, however, draw certain conclusions in regard to the original topographical relations of the various cells encountered in the smear by their general conformation and their staining reactions.

The cells of the intermediate zone are characterized by their cornification and the pyknotic condition of their nuclei. They are, as a rule, large squamous, flat, or partly folded cells. Sometimes they have an elongate or elliptic form. When slightly cornified, they may have a purple or pale bluish color. The nucleus is pyknotic and ordinarily stained dark with haematoxylin. It often shows fragmentation with a number of irregularly shaped granules dispersed into the cytoplasm. With the progress of cornification the nucleus may gradually disappear. Totally cornified cells become irregularly folded and are stained intensely red with eosin.

The cells of the outer basal or pavement cell zone are smaller and more compact, usually rounded or oval, with a relatively large and slightly oval nucleus and a rather dense cytoplasm, which stains deep purple or blue. Some of these cells are invaded by leucocytes similar to the type III cells in rodents.

During post-partum, typical outer basal cells are found. They are round or oval, sometimes elongate or navicular, and have a large round or oval nucleus. A large peri- or paranuclear vacuole is on their characteristic features. They also show vacuolization within the cytoplasm, and are stained intensely blue. Numerous outer basal cells are also present in the post-menopause smears.

Cells belonging to the inner basal zone cannot be easily identified in vaginal smears, being insufficiently differentiated. They have a relatively large, normal nucleus and a very small amount of cytoplasm. Such cells may appear in smears, but their definite identification is very difficult.

The cells of the superficial zone are more differentiated, more variable in size and form, and more deformed, depending upon the extent of differentiation, growth, or degeneration of the superficial zone. Most of the superficial cells are of the ordinary squamous type with flattened or partly folded form and irregular outline. Their nucleus varies greatly in size and is often folded or irregularly shaped. Sometimes it stains faintly, as if it were in a process of gradual resorption; at other times it is surrounded or adjoined by a vacuole. The usual color of the cells varies between purple and blue. Under certain conditions, some of the cells are greatly deformed and display very irregular and atypical forms and partial fragmentation. Some of the cells are invaded by bacteria. Others contain coarse cytoplasmic granules, some in contact with the nucleus.

Many cells have higher differentiation forms and their topographical relations to the various zones are somewhat uncertain. They may be elongate, elliptic, or navicular. Their nucleus is shrunken, slightly elongate, and lies eccentrically, often close under the cellular surface. The interior of the cells is very scantily supplied with cytoplasm, which is often vacuolated. Its periphery is covered with a heavy membrane. Some cells of this type have a round or oval form. 
A very typical and characteristic differentiation of navicular cells occurs during advanced stages of pregnancy. The nucleus of these cells is large in relation to normal cells and their contour is heavier. Their coloration is dark purple or blue.

Papanicolaou ended his 1933 paper with 19 paragraphs summarizing his conclusions, including various photomicrographs of epithelial cells - some of which appeared as watercolor illustrations to emphasize their hues. The most pertinent paragraph follows from Papanicolaou (1933) [5]:

Paragraph 3. The specimen of vaginal fluid, used for the preparation of the smears, was taken with the aid of a glass pipette, and was promptly fixed in a mixture of equal parts of $95 \%$ alcohol and ether. Drying of the smears was carefully avoided. After their fixation the smears were stained with haematoxylin, eosin, and waterblue. The staining method secures a sharp differentiation of all cornified elements.

It is noteworthy that Papanicolaou's 1933 paper focused mainly on endocrinological entities assessed through benign epithelial cell cornification revealed through staining. Papanicolaou was using smears from "various pathological conditions" to contrast against the predominating backdrop of benign, typical cytomorphology. Since the 1928 Battle Creek presentation, cervical cancer was not his preoccupation, at least not officially. His report aimed to describe advances in normal squamous cell maturation assessment using his staining method.

\section{6}

Papanicolaou's staining technique facilitated additional inroads for the vaginal fluid smear method. Based on improved visualization and classification of squamous epithelial cells, he and Shorr developed a method to manage menopause syndrome in patients administered ovarian follicular hormone for the relief of symptoms. This key achievement was based on the cytologic quantification and qualification of stained squamous epithelial cell types reflecting hormone levels. Papanicolaou correlated squamous parabasal cells with degrees of epithelial atrophy, concluding that such cells would mature into superficial types after hormonal treatment. He thus introduced the concept of the "postmenopausal smear." Through these measurable parameters, vaginal epithelium restoration was manageable with strategic hormone administration. These novel clinical achievements in conjunction with alcoholic fixation, and histologic correlations with supporting photomicrography, were reported in the American Journal of Obstetrics and Gynecology in 1936

Inception and Development of the Pap

Stain Method (in a paper entitled "The Action of Ovarian Follicular Hormone in the Menopause, as Indicated by Vaginal Smears") [19].

\section{9}

The year 1939 proved to be pivotal in Papanicolaou's life and career. He had ended his collaborative work with Shorr, although he continued his research on sex hormones in guinea pigs. Following the death of Stockard in 1939, Dr. Joseph C. Hinsey was appointed Head of the Department of Anatomy at Cornell. Papanicolaou approached Hinsey seeking permission to accept a grant from a pharmaceutical firm to finance research in guinea pig steroidal endocrinology. After reviewing Papanicolaou's portfolio, Hinsey surprised Papanicolaou and advised him to decline the grant and instead relaunch his work on the detection of early cervical cancer through the vaginal smear method. Hinsey foresaw a tangible clinical application. Realizing also that Papanicolaou needed access to patients and ample clinical material and a collaborator knowledgeable in gynecological pathology, Hinsey arranged Papanicolaou's collaboration with gynecologist Dr. Herbert F. Traut of the New York Hospital. The combination of Hinsey's visionary decision making and the teamwork between Papanicolaou and Traut revitalized the revolutionary work that transformed the experimental vaginal fluid method into the clinical Pap test by 1954 $[2,8,10,15]$.

Other significant concurrences in 1939 were the waning of the Great Depression and increasing governmental funding to encourage initiatives in preventive medicine [20]. Capitalizing on these dynamics, Hinsey secured financial backing from The Commonwealth Fund to support Papanicolaou's work [8]. These events set Papanicolaou decisively upon a new career course in clinical research. The "lost decade" of cytopathology thus ended $[2,8,10,15]$.

\section{1}

In the following 2 years, Papanicolaou and Traut examined numerous cases from healthy women and from those with cervical neoplasia using the Pap stain with alcoholic fixation and aqueous stain reagents. It was from this work both collaborators reached definitive conclusions on the vaginal smears' utility to detect incipient cervical cancer. As the method's diagnostic potential ap- 
peared indisputable, they reported their conclusions in the American Journal of Obstetrics and Gynecology in 1941 (in a report entitled "The Diagnostic Value of Vaginal Smears in Carcinoma of the Uterus") [21]. Their discussion included an endorsement by Joseph Hinsey. However, a concerning challenge became evident to $\mathrm{Pa}$ panicolaou: even though their confidence in the method was firm, the Pap stain remained unsatisfactory. When comparing epithelial cells exfoliating from cervical squamous carcinoma with those of endometrial adenocarcinoma, and histiocytes common in both disease processes, Papanicolaou felt suboptimal cytoplasmic staining precluded the confident distinction between these cell types. The following extract is from Papanicolaou and Traut (1941) [21]:

In some cases there is an excess of cornified cells, indicating a hyperestrin condition. This was observed more frequently in the early stages of malignancy. Cornification is sometimes due to the coexistence of other pathologic conditions like vaginitis or chronic cervicitis, or to the presence of trichomonas which is not uncommon in cases of cervical malignancy.

Relevant to Hinsey's endorsement, Papanicolaou became wary of interpretive pitfalls stemming from suboptimal staining undermining the method's reproducibility. He was convinced the staining protocol needed refinement.

\section{2}

It was in 1942 that Papanicolaou reported his innovations to upgrade the performance of the Pap stain method. He had discovered that aqueous eosin and waterblue cytoplasmic staining were the source of the problems. He reported these revelations solely in a short but noteworthy publication in the "Special Articles" section of Science in 1942 (entitled "A New Procedure for Staining Vaginal Smears") [1]:

In the course of a study of vaginal smears conducted in cooperation with Dr. Herbert. F. Traut, of the Department of Gynecology of Cornell Medical College and of New York Hospital, for the purpose of diagnosing malignant tumors and other pathological conditions of the female genital tract, it was realized that certain modifications and improvements in our procedure of staining vaginal smears were necessary. Methods which proved to be successful in other applications were not found to be entirely satisfactory in this particular work because of a common disadvantage. The staining of the cells was too deep to permit a sharp definition of their outlines in smears that were relatively thick or contained much blood.
We interpret his use of the term "deep" to mean opaque or obscure.

The following is also from Papanicolaou (1942) [1]:

In most cases of carcinomas and in many other pathological conditions there is a profuse vaginal discharge frequently mixed with blood which forms a heavy film on the slides. In such rich and bloody smears there is considerable crowding and overlapping of cells which, when deeply stained, cannot be well differentiated. This applies more particularly to the small endometrial cells which are often found in menstrual and other uterine bleedings and have a pathognomonic value in the diagnosis of adenocarcinomas of the fundus and of other gynecological conditions. These cells also have great importance in the evaluation of the normal menstrual cycle, of sterility and of estrogenic and other endocrine therapy.

After long experimentation it was found that a much greater transparency and an equally good color differentiation of the cells can be obtained by the use of solutions and stains in $95 \%$ alcohol instead of aqueous solutions. Various alcoholic stains were thus developed, but here only two will be described which are now being used more generally in our laboratory.

The advantages offered by this staining method are the following: (1) The epithelial cells and the erythrocytes are more transparent. Overlapping cells can be more easily differentiated. (2) The color of the acidophilic cells varies from red to orange. This helps in the identification of certain smear types. Basophilic cells stain green to blue-green. (3) Cells or fragments of tissue penetrated by blood take a characteristic orange or orange-green color which permits an easier recognition of small amounts of blood, even when erythrocytes are not distinctly seen. (4) Smears which were subjected to partial or even complete drying can be stained fairly satisfactory. The differential coloring is not entirely lost.

Papanicolaou introduced the terms "acidophilic" and "basophilic" to reflect cornified and noncornified cells, respectively. Ingeniously, he used these terms to establish ranges of hues arising from different physiological states and stained elements based on protein affinities for acidic or basic dyes, including "red to orange," and "green to blue-green," respectively. Papanicolaou achieved this polychromasia by introducing 2 distinct cytoplasmic counter stains into the staining method: orange $(O) \mathrm{G}_{6}$ and either $\mathrm{EA}_{36}$ or $\mathrm{EA}_{25}$. In doing so, the Pap stain protocol then incorporated 5 distinct dyes as EA consisted of light green, Bismarck brown, and eosin. He also adjusted the stain acidity by incorporating specific amounts of phosphotungstic acid (PTA). PTA aimed to intensify the orange color from OG. For instance, he recommended the use of $\mathrm{OG}_{6}$ or $\mathrm{OG}_{8}$ for "normal slides," but $\mathrm{OG}_{5}$ for "a cancer diagnosis" - $\mathrm{OG}_{5}$ would be more acidic, resulting in "a sharper contrast of the abnormal cell types" [1]. The subscripted numerals were a code representing relative color balances and acidity. Papanicolaou discovered that dye uptake by cells was dependent upon reagent $\mathrm{pH}$. These staining phenomena were also dependent upon the 
physical nature of the smears: the degree of fixation, the thickness of the smeared cellular material, the presence or absence of mucus or necrotic material, and the cell types in question. The determinant variables were complex.

Papanicolaou formulated unique stain recipes for optional use depending on anticipated cytomorphology (or staining outcomes) - possibly based on patient clinical history, or even the gross appearance of smeared material. He did not want the staining method to be a limiting factor in his endeavor to secure method acceptance. Interestingly, perhaps to test his methods, he also experimented with the 5-dye method on air-dried cells, and unfixed cells - probably the first ever application of rapid staining for diagnostic purposes in the history of cytopathology. The following is also taken from Papanicolaou (1942) [1]:

Stains EA36 and EA25 can be used for short staining by those who want to make an immediate examination of the slide. No fixative needs to be used. The slides are dipped directly in the staining solution or covered by stain contained in a dropping bottle. The smears are thus fixed and stained simultaneously within a few minutes, although they may be kept in the stain for a longer time without being overstained. The excess stain is washed off in 95\% alcohol and then the slides are carried through absolute alcohol and xylol and mounted in Clarite. The nuclei are stained faintly, but the cells show good differential staining and retain their transparency. Smears stained with this simple method can be restained by a repetition of the procedure described in this paper, including hematoxylin. This will improve the nuclear staining as well as the cellular differentiation and will permit the use of the same smears for more detailed cytologic study.

In the 58th Annual Session of the American Association of Anatomists, held at Cornell Medical College in New York (April 1-3, 1942), Papanicolaou and Traut conducted a microscopy workshop to contrast between normal and malignant cervical epithelial cells, summarized in the abstract to "Cancer Cells in Vaginal Smears" [22]:

Carcinoma of the cervix and of the fundus of the uterus can be diagnosed through the identification of exfoliated cancer cells in vaginal smears. Such cells are demonstrated in microscopic preparations and colored drawings. For comparison, normal cells are also shown.

It is noteworthy that Papanicolaou's reversed approach to use normal cells as cytomorphologic controls along with colored illustrations eventually defined cytopathology. Papanicolaou opted for watercolor drawings to recreate the variable polychromasia then possible through the 5-dye staining method. This heralded the founding of differential and interpretive principles in clinical diagnostic cytopathology possible through strategic staining.

Inception and Development of the Pap Stain Method
Papanicolaou and Traut's conclusions were published in the New York State Journal of Medicine in 1943 (in an article entitled "The Demonstration of Malignant Cells in Vaginal Smears and Its Relation to the Diagnosis of Carcinoma of the Uterus) [23]. Although the authors only briefly referred to the 5-dye Pap stain method in their report, they nonetheless demonstrated the potential diagnostic success possible through its controlled use. The refined staining method then revealed a greater number of cellular elements through transparency, prompting $\mathrm{Pa}$ panicolaou to upgrade his understanding of clinically relevant cytomorphology, leading ultimately to an improved diagnostic confidence. This extract is from that article [23]:

The methods used in making the smears and in staining them have been previously reported. Before one can hope to recognize and analyze the meaning of abnormal cell forms as they appear in vaginal smear preparations, it is necessary to gain experience with the variations in the normal cell pattern as a background.

Papanicolaou used this paper to emphasize 2 case studies on cervical malignancy, both of which were subclinical but had positive cytologic findings. From the improved staining, Papanicolaou reformed his schemata of the diagnostic cytomorphologic features he considered characteristic of cervical squamous carcinoma and those of cervical adenocarcinoma. It was ultimately from the optimized 5-dye staining method that he formulated the application of clinically relevant, cytomorphologic diagnostic criteria. These developments founded the core principles of diagnostic cytopathology, also in nongynecological body fluid practice. In the last statement in their 1943 report, Papanicolaou was poised to raise awareness of these milestones but also of another major work then in progress [23]:

A well-illustrated monograph is in the process of preparation and should be of great assistance to those who desire to learn the exact details of this method of diagnosis.

Papanicolaou and Traut's landmark bound monograph, entitled Diagnosis of Uterine Cancer by the Vaginal Smear, appeared in 1943, published by The Commonwealth Fund [6]. This was undoubtedly a foundational work in gynecological cytopathology.

The reader should note that the book Papanicolaou Revisited by Boon and Chantziantoniou [2] comments on various anecdotal insights surrounding the Pap stain and the 1943 monograph as handed down from Papanicolaou 
to Dr. Mathilde E. Boon through her mentor, Dr. Bernard Naylor [24]. Historically, the monograph proved catalytic for Papanicolaou as it sparked numerous studies by his peers attempting to reproduce his cytologic methods. This resulted in numerous favorable publications securing method validation, and clinical application. Influential reports that also emphasized the effectiveness of the 5-dye staining method were those of Dr. Joe V. Meigs (1947) [25], and Dr. Stuart Lindsay (1949) [26]. The following extract is from Papanicolaou and Traut (1943) [6]:

Various staining procedures have been tried during this study. Most of these were particularly good for the differential staining of the basophilic and acidophilic cells but presented two serious disadvantages. First, they did not stain the nuclei with sufficient differentiation. Good nuclear staining is important in cancer diagnosis because of the significance of morphological changes affecting the nuclei. Second, they stained the cytoplasm of the cells and the erythrocytes rather heavily so that in thick or bloody smears the individual cells could not be well differentiated. This was a great handicap, for in uterine carcinomas the vaginal discharge is usually heavy and mixed with blood. Furthermore, in the adenocarcinomas the search is more difficult because of the small size of the endometrial cells, which when mixed with blood can easily escape attention. On the other hand, when searching for malignant cells, a very thin smear is not particularly desirable.

For these reasons it became necessary to modify our staining methods so as to secure a sharper nuclear staining and a lighter and more transparent cytoplasmic staining without loss of the differential coloring. This was achieved by a prolongation of the time of nuclear staining and by the use of cytoplasmic stains in high alcoholic solutions.

The careful use of a proper staining method is of great importance for one can easily miss an early adenocarcinoma if poor technique is used. Case 5,068 offers an example. This patient was examined in the Gynecological Clinic on December 4, 1940. One smear was taken and was stained by one of the earlier non-transparent methods. No cancer cells or cell groups were noticed. The woman was readmitted four months later and more smears were taken which were stained by one of the newer, more transparent methods. Many typical adenocarcinoma cell groups were found in these smears. The diagnosis was confirmed by curettage. Restaining and reexamination of one of the original smears taken during the first admission have shown the presence of some adenocarcinoma cell groups which were missed during the first examination.

In the 1943 monograph, Papanicolaou announced another modification in the Pap stain protocol defended by the following account [6]:

A slight modification of this method is suggested by Papanicolaou. It consists in the use of stain EA31 instead of EA25 or EA36. Otherwise the staining procedure remains unchanged. Stain EA31 ... seems to stain the basophilic cells somewhat better.

It is thought $\mathrm{EA}_{31}$ produced a better cyanophilic balance due to increased acidity, particularly when analyzing glandular cells arising from adenocarcinoma, to better contrast with squamous epithelial cells that oftentimes appear cornified and thus acidophilic. Papanicolaou again expanded upon the concept of rapid staining, but with another surprising twist [6]:

The task of recognizing cancer cells when present in small numbers, as in early cases, may be facilitated by the omission of counterstaining. Staining of the nuclei with hematoxylin or any other good nuclear stain is sufficient. The cytoplasm of the cells and the blood stain very faintly, and variations in the size and structure of the nuclei can be more readily detected. The same smear can be subsequently counterstained with cytoplasmic stains in order to make a complete study of all cellular elements.

The 1943 monograph incorporated stunning watercolor drawings of cells by Hashime Murayama to support the texts and depict the possible polychromatic outcomes of the revised Pap stain method $[2,6,10]$.

\section{8}

By 1948 Papanicolaou had also acquired substantial experience in nongynecological cytologic practice through application of the refined Pap stain method. Particularly in urinary and gastric specimens, in which suspended epithelial cells may degenerate rapidly, the benefits of the transparent polychromatic staining method proved diagnostically significant. Papanicolaou elected to announce these achievements along with another major work then under way in the American Journal of Public Health in 1948 (the paper was entitled "The Cell Smear Method of Diagnosing Cancer”) [27]:

The salient points of this technique are the immediate fixation of the smears, the avoidance of drying, and a special staining adapted to cancer diagnosis. A good preservation of the structural details of cells and of their nuclei is far more important in cytologic studies than in studies of tissue sections.

It is to be expected that with increasing experience in this new field of cytologic diagnosis our ability to evaluate the many types of cells encountered in the smears will improve considerably. The comparative study of exfoliated cellular elements, normal and abnormal, forms the pivot of our present research activities. An atlas of colored drawings of different types of exfoliated cells is now being prepared in our laboratory.

Use of the smear method in the pathological laboratory in conjunction with other routine diagnostic procedures will undoubtedly result in greater diagnostic accuracy. Furthermore, with the general introduction of this method as a public health measure, it is our hope that the number of operable cases of carcinoma will be markedly increased. 


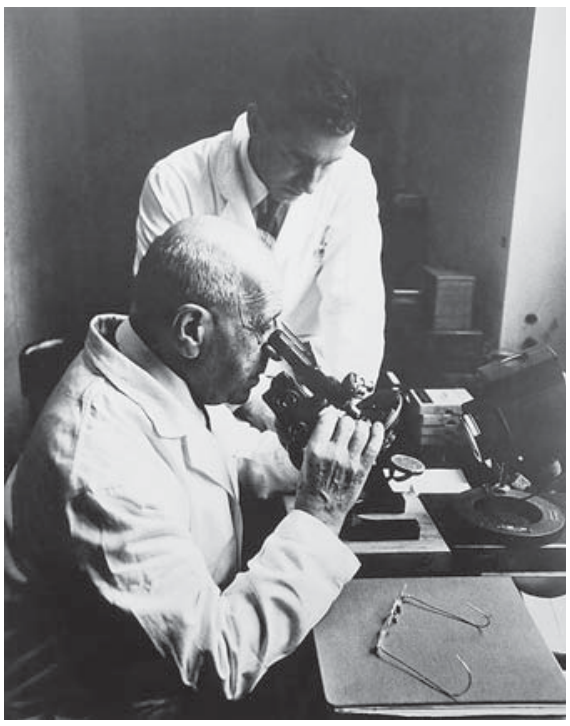

Fig. 1. Dr. George N. Papanicolaou and Dr. John F. Seybolt at Cornell Medical College (undated photograph; courtesy of Dr. R. Marshall Austin).

1954

The Atlas of Exfoliative Cytology published by The Commonwealth Fund in association with Harvard University Press in 1954 is unquestionably Papanicolaou's masterpiece work (Fig. 1-3) [7]. Papanicolaou introduced yet another modification to the Pap stain protocol as described on page 6 of the Atlas. He had concluded that $\mathrm{OG}_{6}$ and $\mathrm{EA}_{36}$ formed the best overall combination of stain dyes for the types of specimens analyzed in general cytopathologic practice, although he also suggested that $\mathrm{EA}_{50}$ may suitably substitute $\mathrm{EA}_{36}$. He had produced sufficient publications by 1954 to amply facilitate the tailoring of dye combinations by his peers for their clinical experimentations and preference.

1960

A supplemental note inserted in the Atlas, entitled "Memorandum of Staining," was also produced by The Commonwealth Fund in 1960 reflecting Papanicolaou's last modification to the Pap stain method [7]. He then recommended the use of $\mathrm{EA}_{65}$ to give improved overall cytoplasmic polychromasia for most cell types in routine practice. Papanicolaou continued to experiment with the staining protocol even after his retirement from Cornell

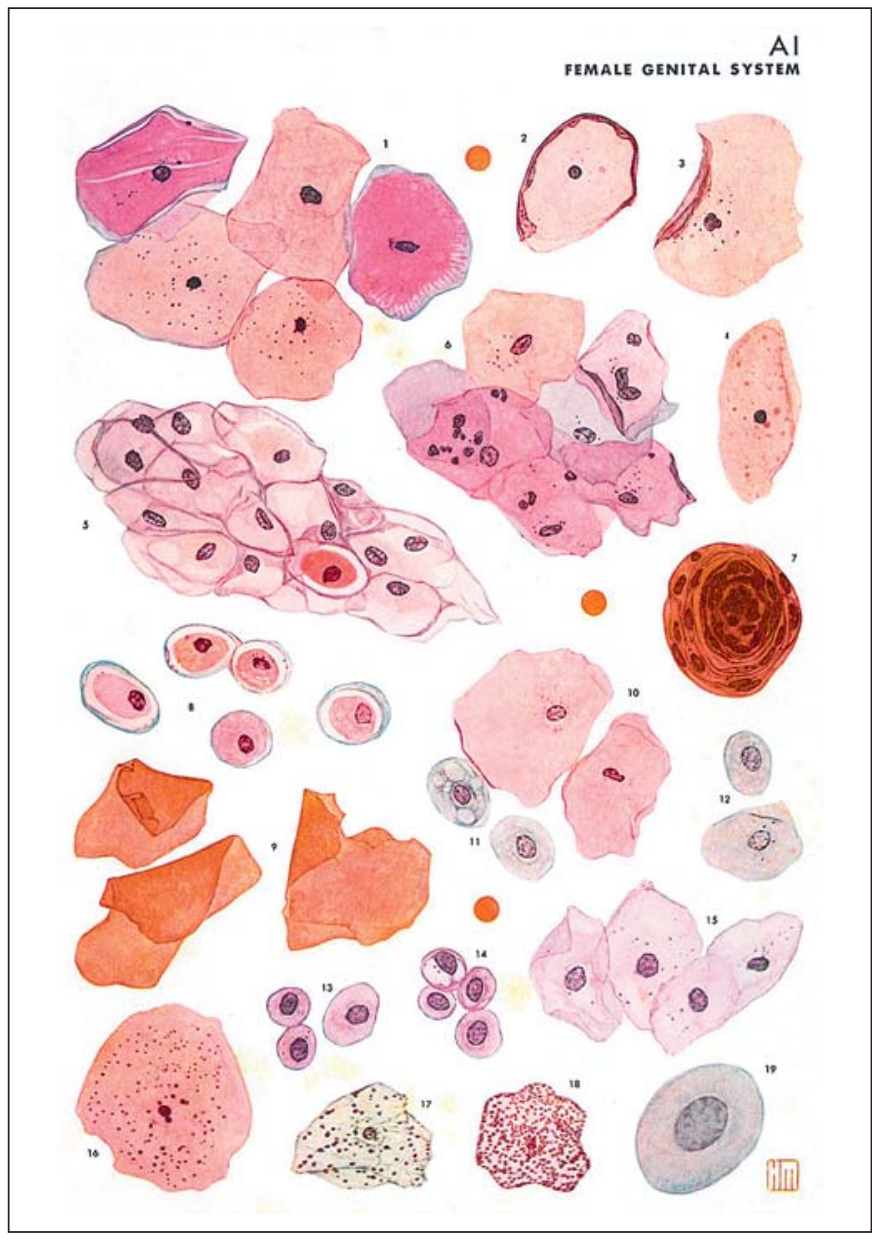

Fig. 2. Plate AI in Atlas of Exfoliative Cytology: "non-malignant squamous epithelial cells found in vaginal and cervical aspiration or swab smears from normal women" [7]. Reprinted with permission from The Commonwealth Fund.

Medical College, as professor emeritus, in 1957, after which he collaborated in the development of Acta Cytologica.

\section{Conclusions}

Dr. George N. Papanicolaou died of myocardial infarction on February 18, 1962. He left behind an astounding legacy of painstaking work that ultimately established the discipline of diagnostic cytopathology. Cytologists can attest to the significance of the refined 5-dye Pap stain method in all facets of modern practice. However, with commercially manufactured staining reagents now widely available, the biochemical characteristics of the stain 


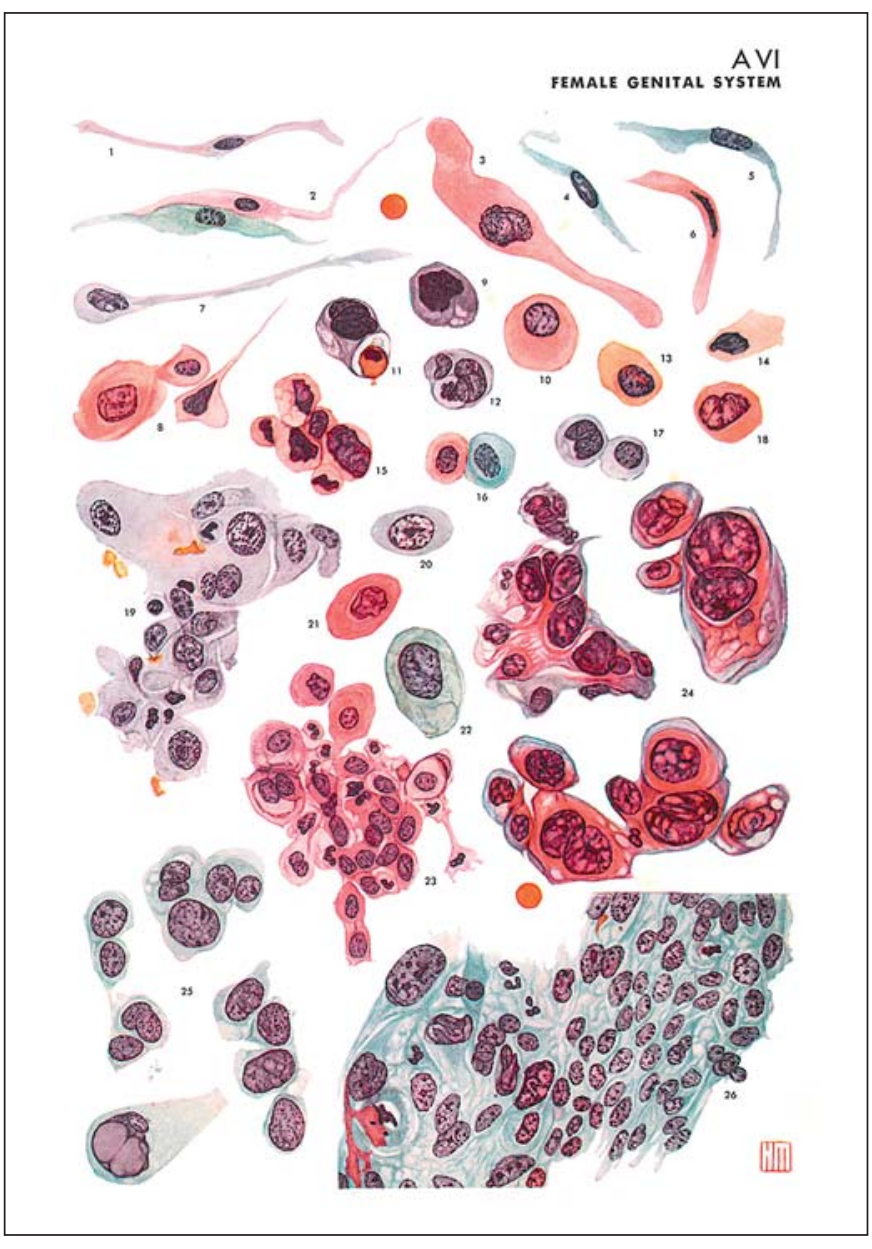

Fig. 3. Plate AVI in Atlas of Exfoliative Cytology: "cells found in vaginal and cervical smears from early and advanced cases of malignancy of the female genital organs" [7]. Reprinted with permission from The Commonwealth Fund.

may be underappreciated for cytologists who might attempt to troubleshoot problematic staining patterns in the clinical setting. This review aimed to document the milestones that led to this tailored staining method and raise awareness of its fundamentals.

It is reasonable to also consider various other circumstances that may have influenced the evolution of the Pap stain. One factor may have been the microscope. Older photographs of Papanicolaou working in his Cornell laboratory reveal his use of a simple monocular microscope equipped with an adjustable mirror directing light into the instrument. Staining practice aside, it is possible optical limitations in pre-World War II microscopes may have spurred the development of cytoplasmic transparency to improve cellular visualization.
Papanicolaou's lack of formal training in surgical pathology may have been a positive factor $[2,8]$. Joseph Hinsey had considered this, foreseeing a major vulnerability for the extensive research work necessary to establish the clinical vaginal smear method. Hinsey's effort to partner Papanicolaou with Herbert Traut was hence astute. Not being a pathologist, Papanicolaou had no occupational attachment to surgical biopsies, formalin fixation, or HE staining. This may also explain his uninhibited experimentation with unconventional staining practices.

The reader should note that in the book entitled Routine Cytological Staining Techniques, Boon and Drijver [3] brought additional insights to light concerning Papanicolaou's experimentation with staining dyes. He intuitively tailored the staining method so that colors demarcated end-points of the human menstrual cycle: its onset, point of ovulation, and completion. The rhythmicity of Andromache's cycles certainly allowed him the means to pinpoint these parameters precisely. As intermediate squamous cells typically stain blue, and pink-red superficial squamous cells predominated at about day 14 of her cycles, a particular combination of cell type and color marked peak estrogen activity. However, although the use of hematoxylin, eosin and waterblue in the earlier versions of the stain method was satisfactory for basic cytology, an associate of Papanicolaou (Mrs. Dorothy Fuller) $[28,29]$ suggested he consider modifications in the Masson's trichrome stain to permit a wider coloration to possibly reveal greater cytologic detail. It is speculated that this sparked Papanicolaou's interest in various combinations of dyes containing acidifying agents that ultimately led to the specific 5 dyes and protocol reported in 1942.

Papanicolaou produced a technical account of his experimentations in the Journal of Laboratory and Clinical Medicine in 1941 (in a paper entitled "Some Improved Methods for Staining Vaginal Smears") [28]. In his report he emphasized the need of a simple and relatively rapid staining method, arguing that cumbersome staining alternatives would be counterproductive:

After long experimentation with over 400 combinations of various imported and domestic stains, a number of satisfactory preparations were developed, some of which are described here. These preparations offer an advantage in that they can be modified to some extent for the purpose of obtaining suitable differentiation of one or another cell type.

The various chemistries employed in the Pap stain method, distinguished by subscripted numeric codes, reflected different recipes of various amounts of powder dye material. Papanicolaou stated the weights of compo- 
Table 1. The salient developmental phases of the 5-dye Pap staining method correlating time frames with cell fixation and nuclear and cytoplasmic staining practice

\begin{tabular}{|c|c|c|c|}
\hline Years & \multicolumn{3}{|l|}{ Pap stain } \\
\hline $1933-1942$ & $95 \%$ alcohol/ether & hematoxylin & aqueous eosin and waterblue \\
\hline \multicolumn{4}{|l|}{$1942-1960$} \\
\hline 1942 & $95 \%$ alcohol/ether & hematoxylin & alcoholic $\mathrm{OG}_{6}$ and $\mathrm{EA}_{36}$ (light green, Bismarck brown, eosin), PTA \\
\hline 1960 & $95 \%$ alcohol & hematoxylin & alcoholic $\mathrm{OG}_{6}$ and $\mathrm{EA}_{65}$ (light green, Bismarck brown, eosin), PTA \\
\hline
\end{tabular}

nent dye powders needed for the varied chemistries and the recommended solution $\mathrm{pH}$ to facilitate the expected color outcomes. However, details of his strategy for allocating the subscripted codes were not specified in his publications.

It is noteworthy that, according to Boon [29], Papanicolaou aimed to produce variable color reactions in the less keratinized squamous cells. As squamous cells mature, their cytoplasm becomes increasingly dense due to an expanding matrix of disulfide bonds linking proteins; therefore, with competing dyes, the smallest dye molecules penetrate and stain cytoplasmic proteins first. With increasing molecular size, the Pap stains' dyes sequence is as follows: (a) OG, (b) eosin, and (c) light green. Therefore, OG stains highly keratinized cells yellow first; then eosin stains superficial cells red; and light green stains parabasal cells green. As hematoxylin prestains cells blue-purple, the latter squamous cells produce turquoise colorations. PTA has 2 important effects: (a) it influences the $\mathrm{pH}$ of the stain reagents, and (b) promotes the staining of light green. As such, the color balance of EA is manipulated by PTA. Finally, a lower solution $\mathrm{pH}$ supports peak staining for light green, and a higher $\mathrm{pH}$ supports peak staining for eosin. Boon and Drijver [3] emphasize the importance of PTA in the Pap stain method. Also, as the staining effects of Bismarck brown are doubtful, this dye is usually omitted in commercially prepared Pap stain platforms $[4,29]$.

As epithelial cells entrapped in vaginal fluids desquamate naturally, their degree of degeneration is typically increased relative to that of more viable cells mechanically removed from cervical epithelia using spatulas or brooms. Therefore, cellular degeneration in the vaginal fluid smears and its associated staining reactions may have been another factor prompting Papanicolaou to secure cellular transparency.

Nonetheless, a definite variable must have been the misinterpretation of cancer cells. In an editorial published in 1958, entitled "Historical Development of Cytology as a Tool in Clinical Medicine and in Cancer Research," $\mathrm{Pa}$ panicolaou produced the following autobiographical reflection 1 year following his retirement from Cornell [30]:

It was in 1940 that I first realized the urgent need for a method of staining that would be more sensitive than the aqueous solution of eosin and waterblue which was used until that time in our laboratory. In reviewing smears from cases of carcinoma of the cervix and adenocarcinomas of the endometrium, which were interpreted and reported as negative, I noticed that in many instances malignant cells, though present in some of the smears, were missed during the first examination because of the lack of translucence which was due to the deep staining of the cell and other components of the smear. An intensive search was thus set in motion, which lasted for approximately two years. During this time a new stain or staining procedure was tested practically every day in our laboratory to the despair of my faithful assistants who finally lost their patience and threatened a sit-down strike unless this nonsense stopped... It surprises me that these stains are still in use because my work was entirely empirical and I thought that by this time new revolutionary methods would have been devised by cytochemists and other investigators better qualified for such work than myself.

Table 1 summarizes the phases of Pap stain development. Until 1933, Papanicolaou analyzed squamous epithelial cell morphology in guinea pigs and later in human vaginal fluid samples using HE staining with limited color reactions, correlating cell type morphology with endocrinology and histology. The metamorphosis of the 5-dye Pap stain method transitioned through 2 salient historical phases. The first was 1933-1942, during which alcohol-ether fixation and aqueous waterblue staining 
was introduced to make cells transparent, aiding the distinction between cervical cancer cells and benign cells with the enhanced quantitative and qualitative assessment of squamous cell maturity. The second was 19421960, when progressively refined alcoholic cytoplasmic counterstaining variations using OG and EA (light green, Bismarck brown, eosin, and PTA) improved cellular transparency, producing wider ranges of polychromasia, optimizing cell type differentiation and diagnostic confidence.

\section{Acknowledgments}

Special thanks are extended to Dr. R. Marshall Austin and the library staff at the University of Pittsburgh Medical Center for identifying rare, difficult to obtain, otherwise unavailable publications and archival material related to Dr. George N. Papanicolaou in support of this work. The corresponding author is personally indebted.

\section{Disclosure Statement}

The authors declare no conflicts of interest arising from this work. No financial support was received from any source.

\section{References}

1 Papanicolaou GN: A new procedure for staining vaginal smears. Science 1942;95:438-439.

2 Boon ME, Chantziantoniou N: Papanicolaou Revisited. Leyden, Coulomb, 2013.

3 Boon ME, Drijver JS: Routine Cytological Staining Techniques. London, MacMillan Education, 1986.

4 Gill GW: Cytopreparation - Principles and Practice. New York, Springer, 2013.

5 Papanicolaou GN: The sexual cycle in the human female as revealed by vaginal smears. Am J Anat 1933;52:519-637.

6 Papanicolaou GN, Traut HF: Diagnosis of Uterine Cancer by the Vaginal Smear. New York, The Commonwealth Fund, 1943.

7 Papanicolaou GN: Atlas of Exfoliative Cytology. New York, The Commonwealth Fund, Harvard University Press, 1954.

8 Carmichael DE: The Pap Smear - Life of George N. Papanicolaou. Springfield, Charles Thomas, 1973.

9 Chantziantoniou $\mathrm{N}$ : The wars against cervical cancer. JASC 2014;3:275-279.

10 Chantziantoniou N: The Pap test - celebrating 100 years in the making and beyond. JASC 2014;3:143-150.

11 Chantziantoniou N: Lady Andromache (Mary) Papanicolaou: the soul of gynecological cytopathology. JASC 2014;3:319-326.

12 Traut HF: Necrologia: Obituary for George N Papanicolaou, MD, PhD. Oncology 1963;16: 351-353.
13 Stockard CR, Papanicolaou GN: A rhythmical 'heat period' in guinea pigs. Science 1917;46: 42-44.

14 Stockard CR, Papanicolaou GN: The existence of a typical oestrous cycle in the guineapig - with a study of its histological and physiological changes. Am J Anat 1917;22:225283.

15 Austin RM, Chantziantoniou N: George Nicholas Papanicolaou: the unlikely story of an intellectual immigrant; one driven to contribute on a large stage; one who finally succeeded by scientifically inspiring numerous cooperating individuals and organizations. JASC 2014;3:331-334

16 Papanicolaou GN: Diagnosis of early human pregnancy by the vaginal smear method. Proc Soc Exp Biol Med 1925;22:436-437.

17 Papanicolaou GN: New cancer diagnosis; in: Proceedings of the Third Race Betterment Conference. Battle Creek, Race Betterment Foundation, 1928, pp 528-534.

18 Koss LG: Papanicolaou's 100th birthday. Acta Cytol 1983;27:217-219.

19 Papanicolaou GN, Shorr E: The action of ovarian follicular hormones in the menopause, as indicated by vaginal smears. Am J Obstet Gynecol 1936;31:806-831.

20 Turnock BJ: Public Health - What It Is and How It Works. Burlington, Jones \& Bartlett Learning, 2012.

21 Papanicolaou GN, Traut HF: The diagnostic value of vaginal smears in carcinoma of the uterus. Am J Obstet Gynecol 1941;42:193206.
22 Papanicolaou GN, Traut HF: Cancer cells in vaginal smears. Anat Rec 1942;82:488.

23 Papanicolaou GN, Traut HF: The demonstration of malignant cells in vaginal smears and its relation to the diagnosis of carcinoma of the uterus. NY State J Med 1943;43:767-768.

24 Naylor B: The century for cytopathology. Acta Cytol 2000;44:709-725.

25 Meigs JV: The vaginal smear - practical applications in the diagnosis of cancer of the uterus. J Am Med Assoc 1947;133:75-78.

26 Lindsay S: The Papanicolaou-Traut method of cancer diagnosis - its use as a routine pathologic laboratory procedure. Calif Med 1949;70:413-416.

27 Papanicolaou GN: The cell smear method of diagnosing cancer. Am J Public Health Nations Health 1948;38:202-205.

28 Papanicolaou GN: Some improved methods for staining vaginal smears. J Lab Clin Med 1941;26:1200-1205.

29 Boon ME: Routine cytologic staining procedures; in Wied GL, Keebler CM, Koss LG, Reagan JW (eds): Compendium on Diagnostic Cytology, ed 6. Chicago, Tutorials of Cytology (IAC), 1990, pp 579-584.

30 Papanicolaou GN: Historical development of cytology as a tool in clinical medicine and in cancer research. Acta Unio Int Contra Cancrum 1958;14:249-254. 